\title{
Replacing the suppressed hormone: toward a better treatment for iron overload in $\beta$-thalassemia major?
}

\section{Domenico Girelli and Fabiana Busti}

Department of Medicine, Section of Internal Medicine, University of Verona, EuroBloodNet Referral Center for Iron Metabolism Disorders, Azienda Ospedaliera Universitaria Integrata of Verona, Verona, Italy

E-mail: DOMENICO GIRELLI - domenico.girelli@univr.it

doi:10.3324/haematol.2020.253393

T he human body lacks a regulatory mechanism able to excrete excess iron. Therefore, any condition increasing iron entry into the body inevitably results in toxic iron overload. ${ }^{1}$ The majority of iron overload disorders can be viewed as endocrine diseases ${ }^{2}$ caused by insufficient production or activity of hepcidin, the key hormone that finely tunes systemic iron homeostasis. ${ }^{3}$ Hepcidin controls body iron content by negatively modulating the absorption of dietary iron from the gut, ${ }^{4}$ and also regulates iron fluxes among different cells and tissues, e.g. from iron-recycling splenic macrophages to erythroid progenitors in the bone marrow. ${ }^{5}$

Deficiency of this hormone, leading to intestinal iron hyperabsorption, is particularly relevant in the pathogenesis of hereditary hemochromatosis, due to gene mutations impairing hepcidin production, but it is also paramount in several inherited "iron-loading" anemias, ${ }^{6}$ par- ticularly in non-transfusion-dependent thalassemias (NTDT), ${ }^{7}$ In these conditions, soluble factors produced by erythroid progenitors during expanded/ineffective erythropoiesis, ${ }^{8}$ including erythroferrone, ${ }^{9}$ directly suppress the synthesis of the hormone in the liver.

Things are more complicated in transfusion-dependent thalassemias (TDT), in which most of the abnormal iron accumulation derives from regular red blood cell transfusions, typically every 2-3 weeks. ${ }^{10}$ Indeed, in TDT, hepcidin level fluctuates according to suppression of erythropoiesis by transfusions, with relatively high and low values immediately after and before red blood cell administration, respectively. ${ }^{11}$ Thus, increased iron absorption can also contribute to iron overload in TDT, at least during intervals between transfusions.

As for many endocrine disorders, a logical therapeutic approach would be the replacement of the missing hor-
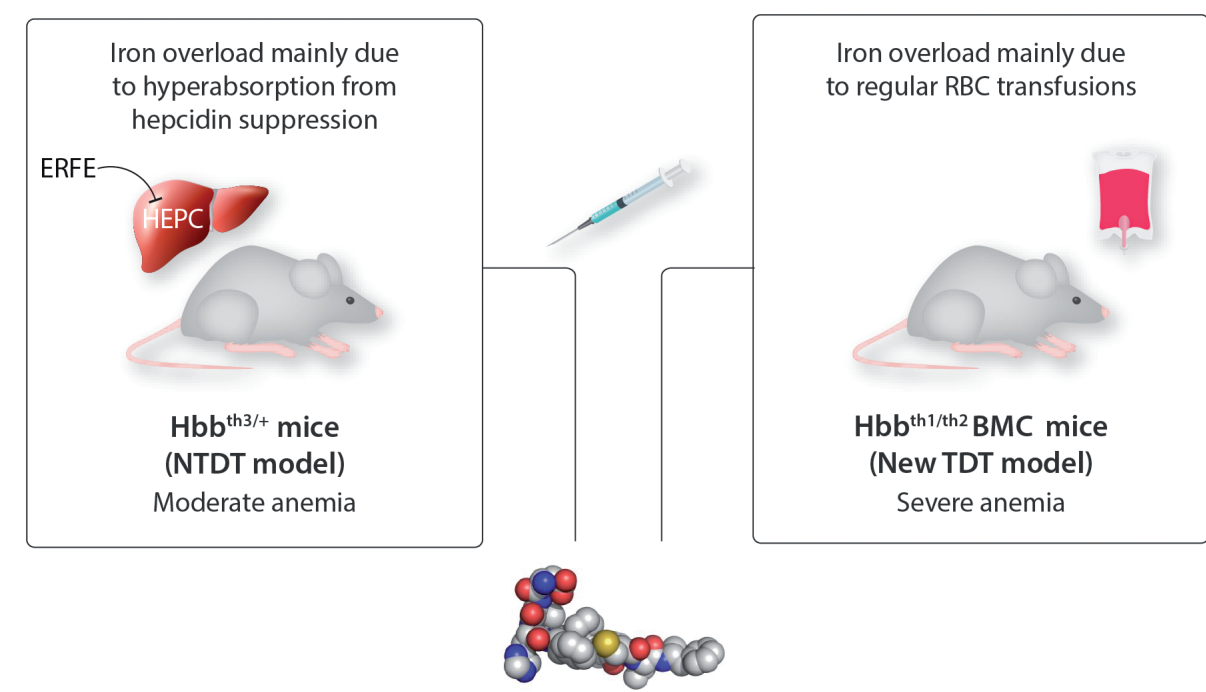

Minihepcidins

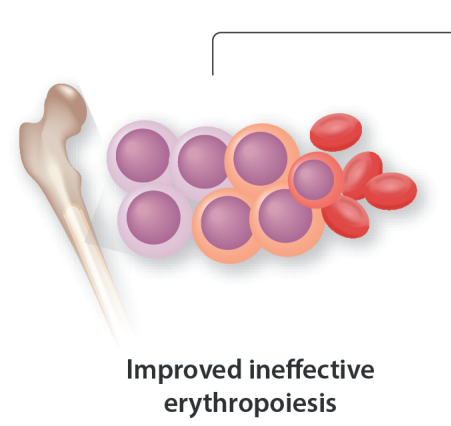

$\uparrow \mathrm{RBC}$ and $\mathrm{Hb} ; \downarrow$ Ret; $\downarrow$ ERFE

$\downarrow$ hemichromes and ROS

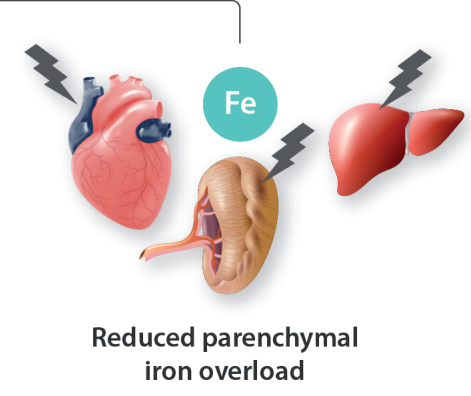

$\downarrow$ iron content in liver, spleen and heart
Figure 1. Effects of minihepcidins in non-transfusiondependent and transfusiondependent $\beta$-thalassemia mouse models. Treatment with minihepcidins has similar beneficial effects in both the nontransfusion-dependent $\beta$-thalassemia mouse mode $\left(\mathrm{Hbb}^{\text {th } 3 /+}\right)$ and in the novel transfusion-dependent mode ( $\left.H b b^{\text {th1/th2 }} B M C\right)$ developed by Casu et al. In particular, in the transfused mice, minihepcidins improved ineffective erythro poiesis and splenomegaly, and reduced the severity of parenchymal iron overload. ERFE: erythroferrone; HEPC: hepcidin; NTDT: non-transfusion-dependent $\beta$-thalassemia; RBC: red blood cells; BMC: bone marrow chimera; TDT: transfusion-dependent $\beta$-thalassemia; $\mathrm{Hb}$ : hemoglobin; Ret: reticulocytes; ROS: reactive oxygen species. 
mone through exogenous preparations. Unfortunately, replacing hepcidin is not as easy as in the case, for example, of levothyroxine in hypothyroidism, or different insulin preparations in type 1 diabetes mellitus. Hepcidin is a small peptide mainly produced by the liver, consisting of only 25 amino acids including eight cysteines which form four disulfide bonds determining a highly folded structure..$^{12}$ Attempts to synthesize a sufficient amount of the hormone in its natural conformation have proven exceedingly difficult. ${ }^{13}$ Moreover, the natural hormone has a short plasma half-life, being rapidly eliminated by proteolysis and renal clearance. ${ }^{14}$

An alternative approach is represented by the production of long-acting molecules, collectively called "minihepcidins". ${ }^{15}$ Minihepcidins are synthetic peptides containing the minimal $\mathrm{N}$-terminal sequence $(7$ to 9 amino acids) of hepcidin still able to bind ferroportin and induce its degradation, further engineered to be resistant to proteolysis. Minihepcidins have been used previously in mouse models of severe hemochromatosis (with knockout of hepcidin anti-microbial peptide; $\left.H A M P^{-/}\right),{ }^{16}$ and NTDT $\beta$-thalassemia. ${ }^{17}$ In the latter model, minihepcidins proved useful in reducing iron overload and splenomegaly, but also improved anemia by either decreasing ineffective erythropoiesis or increasing red blood cell lifespan through reduced formation of hemichromes and reactive oxygen species ${ }^{17}$ (Figure 1).

In this issue of Haematologica, ${ }^{18}$ Casu and colleagues present the first mouse model of TDT $\beta$-thalassemia available so far. Previous attempts to model this disease were hampered by the early death of the animals. To resolve this problem, using an elegant approach the authors intercrossed two previous NTDT strains ( $H b b^{\text {tht } / \text { th }}$ and $\left.H b b^{\text {thr/ }}\right)$ and then transplanted $H b b^{\text {tht } / \text { ht }}$ fetal liver cells into irradiated recipients to obtain $H b b^{\text {tht tht }}$ bone marrow chimera $(B M C)$. These mice showed a severe phenotype resembling $\beta$-thalassemia major, requiring red blood cell transfusions for survival. As in the previous NTDT models, the administration of minihepcidins not only reduced splenomegaly and iron overload (especially in the heart, where it is particularly deadly), but also improved erythropoiesis and anemia (Figure 1). The latter effect is likely related to the apparent paradoxical benefit of iron restriction on thalassemic erythropoiesis, through a reduction in the synthesis of heme, which in turn decreases the production of $\alpha$-globin chains and toxic hemichromes in a coordinated manner. ${ }^{19}$

The study by Casu and colleagues does, however, have several limitations. For example, minihepcidin treatment was started simultaneously with the first transfusion, i.e. before the massive iron accumulation that usually occurs in chronically transfused $\beta$-thalassemic patients. Thus, it remains to be demonstrated whether or not minihepcidins could also be beneficial in a setting more closely resembling clinical practice, in which typical TDT patients are kept on balance within acceptable iron overload by using iron chelators, which in turn are far from being optimal and easy to use. ${ }^{20}$ Another limitation is that the $\mathrm{Hb}^{b^{\text {th }} / \mathrm{h} / \mathrm{h}}$ mice were treated with red blood cell transfusions only for a short period ( 6 weeks). Finally, these mice appear to maintain a particularly high level of iron absorption, which may not mirror what happens in chronically transfused $\beta$-thalassemic patients.

Anyway, the question is: are we ready for hepcidin replacement therapy in the clinic? Despite the promising results of Casu and colleagues, it is still too early to say. No human study on minihepcidins is currently underway. It is unknown whether technical or economic issues will hamper the translation of minihepcidins into the clinic. While, in principle, minihepcidins could be bioavailable after oral administration, ${ }^{14}$ studies in animals used intraperitoneal or subcutaneous administration, which is not as convenient as the oral route. Interestingly, one study with a hepcidin analogue, LJPC-401, is ongoing in adult patients with genetic hemochromatosis (https://clinicaltrials.gov/ct2/show/NCT03395704), but results are not yet available. Despite extraordinary advances toward a definitive cure for $\beta$-thalassemia, by either allogeneic hematopoietic cell transplantation ${ }^{21}$ or gene therapy, ${ }^{22}$ much of the disease's burden occurs in low-income populations with limited access to such sophisticated resources, in which red blood cell transfusions and iron chelation remain the mainstay of therapy. ${ }^{23}$ The research by Casu and colleagues provides a proof of concept that hepcidin replacement therapy or hepcidin agonists represent a fascinating and pathophysiologically sound approach ${ }^{24}$ for treating iron overload in a variety of conditions, including iron-loading anemias. ${ }^{25}$ In the near future, we will understand the place of such drugs in the rapidly evolving and exciting scenario of novel anti-anemic drugs, including activin type II receptor agonists ${ }^{26}$ and others. ${ }^{27}$

\section{References}

1. Hentze MW, Muckenthaler MU, Galy B, Camaschella C. Two to tango: regulation of mammalian iron metabolism. Cell. 2010;142 (1):24-38.

2. Pietrangelo A. Hemochromatosis: an endocrine liver disease. Hepatology. 2007;46(4):1291-1301.

3. Ganz T. Systemic iron homeostasis. Physiol Rev. 2013;93(4):17211741

4. Gulec S, Anderson GJ, Collins JF. Mechanistic and regulatory aspects of intestinal iron absorption. Am J Physiol Gastrointest Liver Physiol. 2014;307(4):G397-409

5. Girelli D, Nemeth E, Swinkels DW. Hepcidin in the diagnosis of iron disorders. Blood. 2016;127(23):2809-2813.

6. Camaschella C, Nai A, Silvestri L. Iron metabolism and iron disorders revisited in the hepcidin era. Haematologica. 2020;105(2):260272 .

7. Sleiman J, Tarhini A, Bou-Fakhredin R, Saliba AN, Cappellini MD, Taher AT. Non-transfusion-dependent thalassemia: an update on complications and management. Int J Mol Sci. 2018;19(1).

8. Ganz T. Erythropoietic regulators of iron metabolism. Free Radic Biol Med. 2019;133:69-74.

9. Coffey R, Ganz T. Erythroferrone: an erythroid regulator of hepcidin and iron metabolism. Hemasphere. 2018;2(2):e35.

10. Angelucci E, Barosi G, Camaschella C, et al. Italian Society of Hematology practice guidelines for the management of iron overload in thalassemia major and related disorders. Haematologica. 2008;93(5):741-752.

11. Pasricha SR, Frazer DM, Bowden DK, Anderson GJ. Transfusion suppresses erythropoiesis and increases hepcidin in adult patients with beta-thalassemia major: a longitudinal study. Blood. 2013;122(1): 124-133.

12. Clark RJ, Tan CC, Preza GC, Nemeth E, Ganz T, Craik DJ Understanding the structure/activity relationships of the iron regulatory peptide hepcidin. Chem Biol. 2011;18(3):336-343

13. Jordan JB, Poppe L, Haniu M, Arvedson T, Syed R, Li V, et al. Hepcidin revisited, disulfide connectivity, dynamics, and structure. J Biol Chem. 2009;284(36):24155-24167.

14. Schmidt PJ, Fleming MD. Modulation of hepcidin as therapy for pri- 
mary and secondary iron overload disorders: preclinical models and approaches. Hematol Oncol Clin North Am. 2014;28(2):387-401.

15. Preza GC, Ruchala P, Pinon R, et al. Minihepcidins are rationally designed small peptides that mimic hepcidin activity in mice and may be useful for the treatment of iron overload. J Clin Invest. 2011;121(12):4880-4888.

16. Ramos E, Ruchala P, Goodnough JB, et al. Minihepcidins prevent iron overload in a hepcidin-deficient mouse model of severe hemochromatosis. Blood. 2012;120(18):3829-3836.

17. Casu C, Oikonomidou PR, Chen H, et al. Minihepcidin peptides as disease modifiers in mice affected by beta-thalassemia and polycythemia vera. Blood. 2016;128(2):265-276.

18. Casu C, Chessa R, Liu A, et al. Minihepcidins improve ineffective erythropoiesis and splenomegaly in a new mouse model of adult $\beta$ thalassemia major. Haematologica. 2020;105(7):1835-1844.

19. Camaschella C. Treating iron overload. N Engl J Med. 2013;368(24): 2325-2327.

20. Coates TD. Iron overload in transfusion-dependent patients.
Hematology Am Soc Hematol Educ Program. 2019;2019(1):337-344 21. Angelucci E. Hematopoietic stem cell transplantation in thalassemia. Hematology Am Soc Hematol Educ Program. 2010;2010:456-462.

22. Thompson AA, Walters MC, Kwiatkowski J, et al. Gene therapy in patients with transfusion-dependent beta-thalassemia. N Engl J Med. 2018;378(16):1479-1493.

23. Galanello R, Origa R. Beta-thalassemia. Orphanet J Rare Dis. 2010;5:11.

24. Casu C, Nemeth E, Rivella S. Hepcidin agonists as therapeutic tools. Blood. 2018;131(16):1790-1794.

25. Camaschella C, Nai A. Ineffective erythropoiesis and regulation of iron status in iron loading anaemias. Br J Haematol. 2016;172(4):512-523.

26. Cappellini MD, Viprakasit V, Taher AT, et al. A phase 3 trial of luspatercept in patients with transfusion-dependent beta-thalassemia. N Engl J Med. 2020;382(13):1219-1231.

27. Busti F, Marchi G, Lira Zidanes A, Castagna A, Girelli D. Treatment options for anemia in the elderly. Transfus Apher Sci. 2019;58(4):416-421.

\section{ABL-class fusion positive acute lymphoblastic leukemia: can targeting ABL cure ALL?}

\section{Thai Hoa Tran ${ }^{1}$ and Stephen P. Hunger ${ }^{2}$}

${ }^{1}$ Division of Pediatric Hematology-Oncology, Charles-Bruneau Cancer Center, CHU Sainte-Justine, University of Montreal, Montreal, Quebec, Canada and 'Department of Pediatrics, The Center for Childhood Cancer Research, Children's Hospital of Philadelphia, The Perelman School of Medicine, University of Pennsylvania, Philadelphia, PA, USA

E-mail: STEPHEN HUNGER - hungers@email.chop.edu

doi:10.3324/haematol.2020.252916

$\mathrm{F}$ ive-year survival rates for pediatric acute lymphoblastic leukemia (ALL), a malignancy that was incurable in the 1950s, now exceed $90 \% .{ }^{1}$ However, $15-20 \%$ of National Cancer Institute (NCI) high-risk (HR) B-lineage ALL (B-ALL) patients relapse, and post-relapse outcomes remain poor, particularly following early marrow relapse [5-year overall survival (OS): 28\%]. ${ }^{2}$ Genomic advances have identified a novel B-ALL subtype characterized by a heterogeneous spectrum of kinase-activating alterations, producing a gene expression signature similar to that of Philadelphia chromosome-positive $\left(\mathrm{Ph}^{+}\right) \mathrm{ALL}$, without the canonical BCR-ABL1 oncoprotein, referred to as BCR-ABL1-like ALL or Ph-like ALL, and now recognized as a provisional disease entity in the 2016 World Health Organization's classification of acute leukemias., Ph-like ALL is associated with adverse clinical features and poor outcomes despite modern therapy. ${ }^{4-6}$ It occurs in approximately $15 \%$ of children with NCI HR B-ALL and over $25 \%$ of adults with B-ALL, and contributes disproportionately to relapses. ${ }^{4,6}$ Among Ph-like ALL patients, $10-14 \%$ of them harbor rearrangements of ABL-class genes (ABL1, ABL2, CSF1R, LYN, PDGFRA, PDGFRB) other than $B C R-A B L 1$, collectively representing $2-3 \%$ of pediatric B-ALL cases. ${ }^{4-6}$ While there are anecdotal reports of the short-term efficacy of adding the ABL tyrosine kinase inhibitors (TKI) imatinib or dasatinib to chemotherapy, ${ }^{4,7,8}$ controlled data are lacking regarding the long-term efficacy of this approach. In this issue of Haematologica, Cario et al. ${ }^{9}$ provide important new information on treatment of children with ALL and ABL-class fusions.

They report $46 \mathrm{ABL}$-class fusion positive $\mathrm{B}$-ALL patients (15 involving ABL1, 5 ABL2, 3 CSF1R, and 23
PDGFRB) who were originally enrolled on the AIEOPBFM ALL 2000 and 2009 trials, and identified retrospectively. ABL-class fusion-positive cases had a substantially worse early treatment response than other patients, as reflected by prednisone-poor response $(50 \%$ vs. $5.6 \%$, $P<0.0001)$ or minimal residual disease $(\mathrm{MRD}) \geq 5 \times 10^{-4}$ at end-induction $(71.4 \%$ vs. $19.2 \%, P<0.0001)$ and end-protocol Ib $(51.2 \%$ vs. $5.1 \%, P<0.0001)$. Thirty-six of 46 patients $(78.3 \%)$ were classified as HR (vs. $11.1 \%$ of ALLBFM 2000 B-ALL patients overall), and more than half (25 of $46,54.3 \%$ ) underwent hematopoietic stem cell transplantation (HSCT) in first complete remission (CR1). For the cohort of 46 patients with ABL-class fusions, the 5year event-free survival (EFS) and OS were $49.1 \pm 8.9 \%$ and $69.6 \pm 7.8 \%$, respectively. The 5 -year cumulative incidence of relapse (CIR) and treatment-related mortality (TRM) were $25.6 \pm 8.2 \%$ and $20.8 \pm 6.8 \%$, respectively. Thirteen patients (13 of $46,28.3 \%$ ) received TKI in combination with chemotherapy post-induction; their outcomes were not significantly different from those in the no-TKI group ( $\mathrm{n}=33$ ) (5-year EFS $62.9 \%$ vs. $47.7 \%$, $P=0.98$; 5 -year OS $75.5 \%$ vs. $70.9 \%, P=0.64$ ). In parallel, ABL-class patients treated with or without HSCT had similar outcomes (5-year EFS $47.9 \%$ vs. $55.0 \%, P=0.35 ; 5$ year OS $66.7 \%$ vs. $84.0 \%, P=0.22)$. Notably, in the 33 patients treated without TKI, there was a trend towards lower CIR rate among patients who underwent HSCT $(n=16)$ compared to those who did not $(n=17)(13.2 \%$ vs. $43.8 \%, P=0.06$ ). The TRM rate was, nevertheless, exceedingly high in the HSCT group $(32.3 \%$ vs. $0.0 \%$, $P=0.034)$. Furthermore, the majority of events in the HSCT group were non-relapse events, while relapses predominate in the no-HSCT group. 